\title{
Experimental study of the effect of air injection on the pressure pulsations in the hydro turbine flow path under different operating conditions
}

\author{
Andrey Minakov ${ }^{1,2^{*}}$, Dmitry Platonov ${ }^{1,2}$, Alexandra Maslennikova ${ }^{1}$ and Dmitry Dekterev ${ }^{1,2}$ \\ ${ }^{1}$ Siberian Federal University, 79 Svobodny Ave., 660041 Krasnoyarsk, Russia \\ ${ }^{2}$ Institute of Thermophysics, SB RAS, 1 Lavrentyev Ave., 630090, Novosibirsk, Russia
}

\begin{abstract}
The paper presents an experimental study of oscillatory response in the Francis turbine of hydraulic unit. The experiment was performed on large-scale hydrodynamic test-bench with impeller diameter of $0.3 \mathrm{~m}$. The effect of air injection on the intensity of pressure pulsations was studied at the different regimes in the hydraulic unit. It was revealed that air delivery into the flow path system of the turbine results in almost two-fold reduction of pressure pulsations, but for optimal regimes, there is an increase in pressure pulsations.
\end{abstract}

\section{Introduction}

An important task for HPP is regulation of power in the energy system. During load changing hydraulic units repeatedly undergo off-design modes of operation. Under these flow conditions a significant part of swirl, after passing through the water turbine impeller, is remained. The instability of swirling flow may cause the emergency of intense lowfrequency hydrodynamic fluctuations that threaten the reliability of turbine design [1-3]. Precessing vortex rope is a serious danger for the hydraulic turbine equipment due to powerful flow pulsations that lead to strong vibrations of hydraulic turbine construction and in the case of resonance can lead to destruction of the equipment. Pressure pulsations generated by the vortex rope precession, may also affect cavitations processes, enhancing cavitations erosion.

Up to date, numerous methods are proposed to stabilize flow in the hydraulic units [47]. Each of them has its advantages and disadvantages, and requires further detailed study. In this study, the effect of air delivery on the oscillatory response of the flow was experimentally investigated on the model hydrodynamic stand with Francis turbine.

\section{Experimental techniques}

The model water turbine with radial-axial impeller is part of the hydropower test-bench at the Laboratory of Hydraulics and Hydraulic Machines of the Siberian Federal University see in Fig. 1. Maximum water head in the test-bench is $3.5 \mathrm{~m}$, the diameter of the impeller

* Corresponding author: tov-andrey@yandex.ru 
is $\mathrm{D}=0.3 \mathrm{~m}$. Hydraulic unit includes a pressure tank, performing the functions of the upper pool, power conduit with the diameter of $400 \mathrm{~mm}$, and penstock valve, equipped with electric drive. Water from the penstock enters the spiral chamber and through the guide vanes flows to the runner. Then water through the draft tube gets into the lower pool with glass walls. The width of the pool was equal to 1.52 meters. Diffusion grid is designed to reduce fluctuations in water level before weir gauge. Weir gauge is used for measuring water flow rate and is fixed by water gauge.

After weir gauge, the water is discharged into the collecting tank and then is diverted through pipe into the pool. From the pool, water is pumped into the pressure tank. The water intake in the collecting tank is performed through the suction cap with mesh. The water flow rate is controlled by gate valve with electric actuator. To record the pressure pulses, the test-bench was equipped with a piezoelectric flow rate sensors (type 014MT) designed to convert rapidly varying and pulsed pressure into an electrical signal. The signal from the sensors came through an amplifier (LE-41) and an analog digital converter (ADC E14-140 1-card) to the computer for further processing.
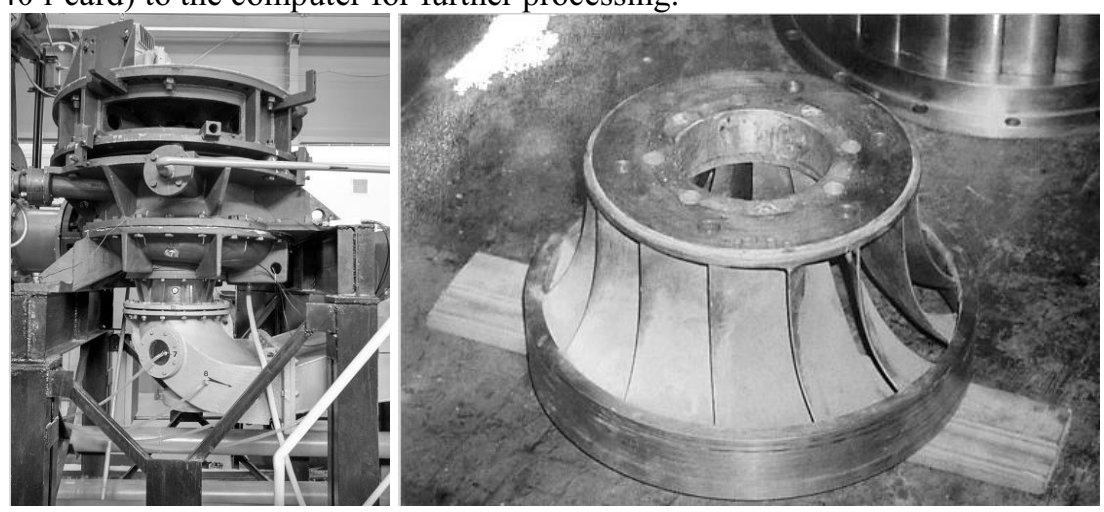

Fig. 1. Model of water turbine with radial-axial impeller.

\section{Experimental results}

In the course of experiments we carried out measurements within a wide range of the hydraulic unit operation both with air and without air. The degree of opening of the guide vanes was varied in the experiment, while the pressure remained constant. Then all the necessary parameters, namely, impeller rotation frequency, the flow rate through the hydraulic unit, and generating current and voltage were recorded.

The behaviour of the frequency and the pressure pulsations intensity in the waterconveyance system of the turbine is of great interest for high-head power plants since the precession of a vortex core is a serious hazard to the water turbine equipment. Pressure pulsations were recorded at specified points during five minutes for each of the operation modes.

Figures 2 and 3 below present the experimental results on time variations of pressure pulsation in the draft tube diffuser carried out for two operating modes of the hydrodynamic stand: maximum pressure pulsation regime at opening of $5 / 15$, and near optimum regime at opening of $8 / 15$. From the figures, indicating the point in time corresponding to air injection into the flow path (red line), it is evident that in a regime with maximum pulsations, a significant decrease is observed in pressure pulsations amplitude, while in the regime near to the optimum one, conversely - pressure pulsations increased. 


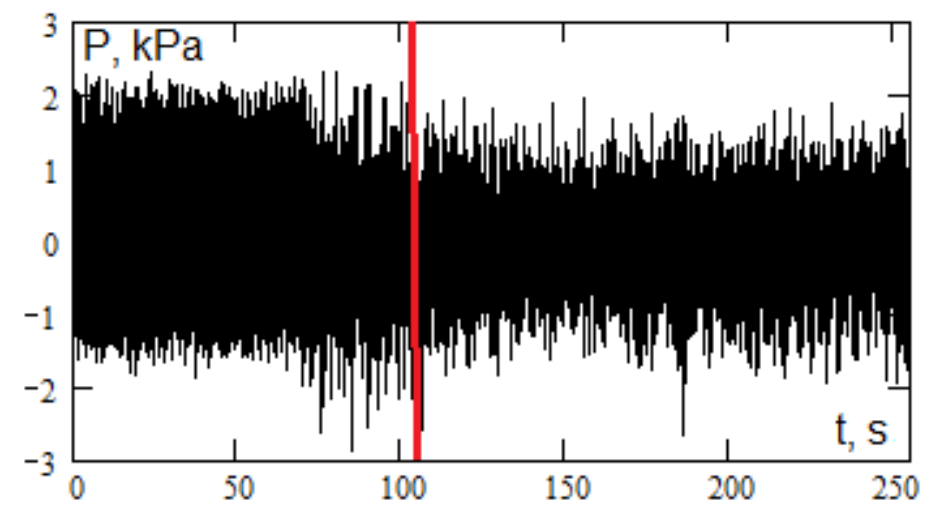

Fig. 2. Time dependence of the pressure pulsation in the draft tube diffuser (opening 5/15).

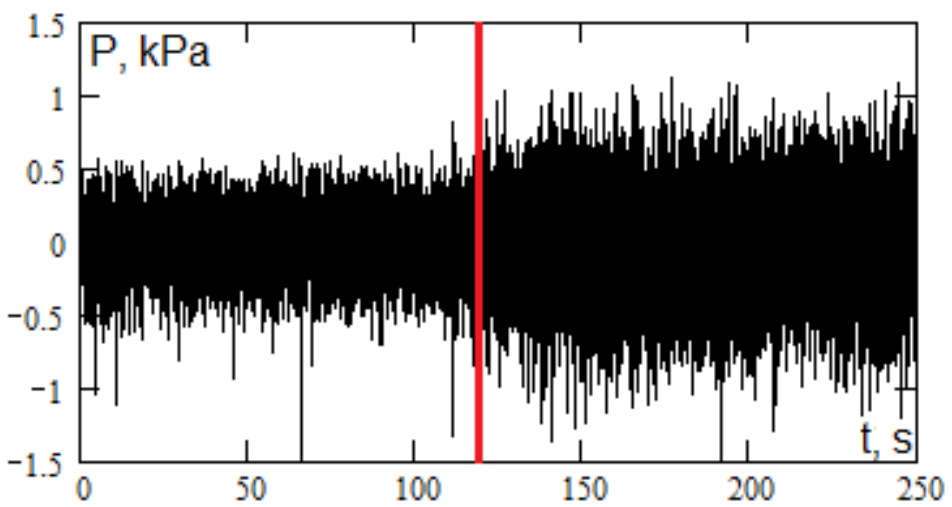

Fig. 3. Time dependence of the pressure pulsation in the draft tube diffuser (opening 8/15).

Figures 4 presents experimental dependence of the pressure pulsation amplitude in the draft tube cone on the opening of the guide vanes. The dependence shows that the air injection allows one to reduce the pressure pulsations in the most dangerous zones by more than 2 times, however, in the region of optimal regimes, the pressure increases. This fact indicates the advisability of air supply only at certain loads on the hydroelectric unit.

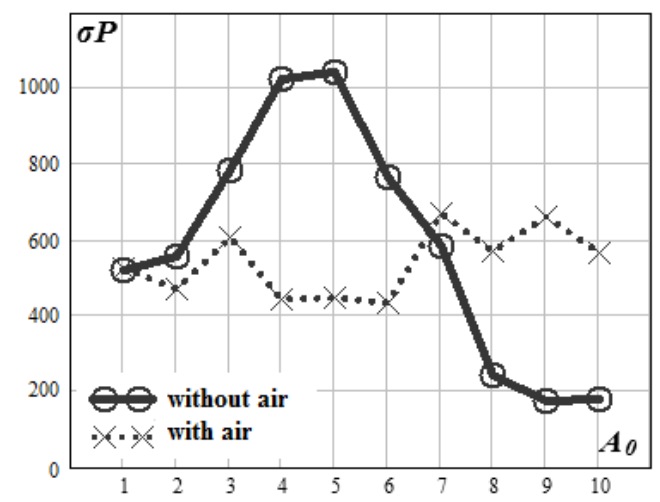

Fig. 4. Dependence of the pressure pulsation amplitude in the draft tube cone on the opening of the guide vanes. 


\section{Conclusion}

The work deals with experimental investigation of pressure pulsations for different flow regimes in the hydraulic unit of the medium-scale hydrodynamic stand with air injection in flow path. The authors obtained correlations between the pressure pulsations and the opening of the guide vanes for constant pressure heads, in the hydraulic turbine taking into account the air phase. Air was supplied into a spiral chamber of the turbine. This method is one of the most promising to reduce loads on the hydraulic unit, caused by the precession of the vortex core in the space behind the runner.

The results of the study have shown that it is possible to significantly reduce the pressure pulsations using air at the maximum pressure pulsations regime. However, this effect needs further investigation. In particular, it is necessary investigating the effect of air injection point.

The current work is performed at partial support of the projects funded by the Russian Foundation for Basic Research and Krasnoyarsk Regional Fund for Support of Scientific and Scientific-Technical Activities (16-41-243081).

\section{References}

1. A.V. Minakov, A.V. Sentyabov, D.V. Platonov, A.A. Dekterev, A.V. Zakharov, Comp. \& Fluids 111, 197 (2015)

2. A.V. Minakov, A.V. Sentyabov, D.V. Platonov, A.A. Dekterev, A.V. Zakharov, Int. J. Heat Fluid Flow 53, 183 (2015)

3. D. Platonov, A. Minakov, D. Dekterev, A. Sentyabov, A. Dekterev, J. Phys. Conf. Ser. 754, 062007 (2016)

4. H.T. Falvey, U.S. Bureau of Reclamation. (1971)

5. T.L. Wahl, Colorado: Colorado State University, (1990)

6. H. Grein, 10th Symposium of the International Association for Hydraulic Research. (1980)

7. S. Bhan, 5th International Symposium on Hydro Power Fluid Machinery. (1988) 